\title{
Gene transfer of the neuronal NO synthase isoform to cirrhotic rat liver ameliorates portal hypertension
}

\author{
Qing Yu, ${ }^{1}$ Rong Shao, ${ }^{1}$ Hu Sheng Qian, ${ }^{2}$ Samuel E. George, ${ }^{2}$ and Don C. Rockey ${ }^{1,3}$
}

${ }^{1}$ Duke Liver Center,

${ }^{2}$ Division of Cardiology, Department of Medicine, and

${ }^{3}$ Department of Cell Biology, Duke University Medical Center, Durham, North Carolina 27710, USA

Address correspondence to: Don C. Rockey, Liver Center Laboratory, Room 336, Sands Building, Box 3083 ,

Duke University Medical Center, Durham, North Carolina 27710, USA. Phone: (919) 684-8727;

Fax: (919) 684-4983; E-mail: dcrockey@acpub.duke.edu.

Portions of this work were presented at the 50th Annual Meeting of the American Association for the Study of Liver Disease in Dallas, Texas on November 5-9, 1999.

Received for publication July 28, 1999, and accepted in revised form February 1, 2000.

Reduced production of nitric oxide (NO) in the cirrhotic liver results from a defect in hepatic endothelial cell nitric oxide synthase (ecNOS) and appears to contribute to the high intrahepatic resistance and portal hypertension typical of cirrhosis. Therefore, we postulated that targeting a heterologous NOS isoform to sinusoidal endothelial cells or other perisinusoidal cells, such as hepatic stellate cells, would counter the defect in NO production and reduce resistance to blood flow. Recombinant adenovirus (Ad) carrying the neuronal NOS gene (nNOS) targeted liver sinusoidal endothelial cells, stellate cells, and hepatocytes more efficiently than the corresponding cells in cirrhotic livers, but transduction rates were substantial even in cirrhotic animals. Expression of nNOS in each liver cell type, whether from normal or injured liver, caused increased NO production and inhibited endothelin-1-induced contractility of perisinusoidal stellate cells. Finally, in 2 different in vivo models of cirrhosis and portal hypertension, transduction of livers with recombinant Ad.nNOS significantly reduced intrahepatic resistance and portal pressure. The data highlight the feasibility of gene transfer to diseased liver and hepatic cells and demonstrate the potential of a novel therapy for portal hypertension caused by cirrhosis.

J. Clin. Invest. 105:741-748 (2000).

\section{Introduction}

The common result of many types of chronic liver injury is cirrhosis, which leads to increased intrahepatic resistance and portal hypertension (1). Portal hypertension in turn has profound clinical consequences, many of which are associated with substantial morbidity and mortality. The pathological basis of portal hypertension is complex and involves multiple factors (2). However, in most instances an increase in intrahepatic resistance to blood flow is an early and critical component. Recent evidence links perisinusoidal stellate cells (also known as Ito cells or lipocytes), which are analogous to tissue pericytes or vascular smooth muscle cells, to a role in portal hypertension via their capacity to regulate blood flow within the liver by contraction and constriction of sinusoids (3-7).

In the injured liver, stellate cells undergo a striking functional transition termed activation. A critical feature of activation is the acquisition by stellate cells of smooth muscle proteins, including smooth muscle isoforms of actin and myosin (8). This transition is associated with an enhanced contractile phenotype; as a result of activation, stellate cell contractility is greatest in the injured liver $(5,9)$. Recent data indicate that the family of endothelins are potent stimulators of stellate cell contraction, whereas vasorelaxing sub- stances including nitric oxide (NO) counterbalance the contractile response. Further, in the injured liver, ET-1 production is increased (as a result of enhanced production by stellate cells [refs. 10-12]), and in addition, NO release from sinusoidal endothelial cells is reduced, ostensibly owing to impaired function of endothelial cell NOS (ecNOS) (13-16). These data indicate that the dynamic balance of ET- 1 and NO is abnormal in cirrhosis, favoring sinusoidal and possibly vascular wall constriction by perisinusoidal stellate cells and perhaps by hepatic vascular smooth muscle cells, respectively. Further, the data suggest that on a cellular level, anomalous production of each ET-1 and NO in the injured liver contributes to elevated intrahepatic resistance and portal hypertension.

Given the apparent defect in ecNOS function in sinusoidal endothelial cells in cirrhotic liver, we aimed to determine whether transduction of sinusoidal cells with a heterologous isoform of NOS (rather than ecNOS itself) was possible, and if so, to understand the physiological effect of its gene transfer into isolated liver cells as well as in the whole liver. In this study, we demonstrate efficient adenovirus-mediated (Ad-mediated) transduction of neuronal NOS (nNOS) in liver sinusoidal (each sinusoidal endothelial and stellate) cells and in hepatocytes both in vitro and in vivo. Addi- 
tionally, expression of nNOS in each cell type led to enhanced NO release, an effect that resulted in inhibition of stellate cell contraction. Finally, gene transfer of nNOS to liver cells after development of cirrhosis led to significant reduction in portal pressure and intrahepatic resistance to flow.

\section{Methods}

Animals and liver injury. Hepatic injury was induced in male retired breeder Sprague-Dawley rats $(450-550 \mathrm{~g})$ by bile duct ligation (BDL) for 9 days or by intragastric administration of carbon tetrachloride $\left(\mathrm{CCl}_{4}\right.$; administered at a concentration of $1 \mathrm{~mL} / \mathrm{kg}$ once per week for 10 weeks), each as described previously $(17,18)$. All animals received humane care according to National Institutes of Health guidelines.

Cell isolation and culture. Hepatocytes, stellate cells, and sinusoidal endothelial cells were isolated as described previously (19-21). For nonparenchymal cells, after in situ perfusion of the liver with $20 \mathrm{mg} \%$ pronase (Boehringer Mannheim, Indianapolis, Indiana, USA) followed by collagenase (Crescent Chemical Co., Hauppauge, New York, USA), dispersed cell suspensions were layered on a discontinuous density gradient of $8.2 \%$ and 15.6\% Accudenz (Accurate Chemical and Scientific, Westbury, New York, USA). The resulting upper layer consists of more than $95 \%$ stellate cells. Endothelial cells in the lower layer were further purified by centrifugal elutriation $(18 \mathrm{~mL} / \mathrm{min}$ flow). Hepatocytes isolated after collagenase perfusion (as just described) were placed in modified medium 199 OR (22) containing 5\% FBS (GIBCO BRL, Grand Island, New York, USA); nonparenchymal cells were grown in the same medium containing $20 \%$ serum (10\% horse $10 \%$ calf) (Flow Laboratories, Inc., Naperville, Illinois, USA). The viability of all cells was verified by phase contrast microscopy and the ability to exclude propidium iodide. Cell viability of cultures used for study was greater than $95 \%$.

Inhibition of NOS in vivo. NOS was inhibited in vivo using 7-nitroindazole (7-NI; Sigma Chemical Co., St. Louis, Missouri, USA), a selective nNOS inhibitor (23). 7-NI was dissolved in solution containing dimethyl sulfoxide/propylene glycol/distilled water (ratio of 1:3:6) and administered by intraperitoneal injection in a volume of $2.0 \mathrm{~mL} / \mathrm{kg}$. 7-NI was injected 1 day before BDL or the last dose of $\mathrm{CCl}_{4}$ at the dose of $25 \mathrm{mg} / \mathrm{kg}$ for a total of 4 doses.

Ad preparation. Ad.nNOS, containing a cDNA-encoding rat neuronal NOS, driven by the cytomegalovirus immediate-early enhancer, was generated as described previously (24). A recombinant Ad encoding $\beta$-galactosidase (Ad. $\beta$-gal), in the same cassette as Ad.nNOS, served as a control virus.

For cellular transduction with Ad, isolated cells were plated at a density of $1 \times 10^{6}$ cells $/ \mathrm{mL}$ in 35 - or $60-\mathrm{mm}$ plastic or collagen-coated (hepatocytes and sinusoidal endothelial cells) culture and exposed to indicated concentrations of Ad for 2 hours at $37^{\circ} \mathrm{C}$. Subsequently, Ad-containing medium was exchanged for standard medium. For in vivo experiments, rats were anesthetized and Ad.nNOS or Ad. $\beta$-gal was administered via the femoral vein at the indicated titer. In these experiments, we timed Ad administration to occur such that gene expression (i.e., 7-10 days after Ad injection) would be present at the time of cell harvest or physiological harvest. For the BDL model, Ad was administered 2 days after BDL; for the $\mathrm{CCl}_{4}$ model, Ad was administered 2 days after the 10th and final dose of $\mathrm{CCl}_{4}$. Cells were routinely harvested (and perfusion experiments performed) 7 days after Ad administration. In preliminary experiments, we found that concentrations of $1.0 \times 10^{10}$ $\mathrm{pfu} / \mathrm{kg}$ to $6.0 \times 10^{11} \mathrm{pfu} / \mathrm{kg}$ effectively transduced high proportions of each parenchymal and stellate and endothelial cells, with small variation in transduction rate. Therefore, we chose a concentration of $1.5 \times 10^{11}$ $\mathrm{pfu} / \mathrm{kg}$ of Ad.nNOS to examine nNOS gene transfer. Heparinized blood was obtained from all rats at the time they were sacrificed.

NADPH-diaphorase activity, immunohistochemistry. To determine transduction efficiency after Ad.nNOS exposure, NADPH-diaphorase activity were assessed. Cells were washed and fixed for 30 minutes followed by permeabilization with $0.3 \%$ Triton X-100 in PBS for another 30 minutes. Finally, cells were stained with $1.33 \mu \mathrm{L} / \mathrm{mL}$ nitroblue tetrazolium and $2 \mathrm{mg} / \mathrm{mL} \mathrm{NADPH}$ in the same buffer for approximately 1 hour until the development of blue-purple staining was observed. Mock-infected cells were stained in parallel for the same period.

Immunohistochemistry to identify nNOS expression was performed as described elsewhere (25). Briefly, liver tissues were fixed in $4 \%$ buffered-paraformaldehyde, embedded in paraffin, sectioned $(6-8 \mu \mathrm{m})$, and deparaffinized by standard methods. After incubation of sections with $10 \%$ sheep serum in PBS with $0.1 \%$ Triton X100 , sections were incubated overnight with monoclonal anti-nNOS antibody (Transduction Laboratories, Lexington, Kentucky, USA), diluted 1:100. The first antibody was viewed using an avidin-biotin complex /alkaline phosphatase substrate detection method (Vector Laboratories, Burlingame, California, USA). Sections were lightly counterstained with Methyl Green (Vector Laboratories), viewed, and photographed with a Nikon TE 300 photomicroscope (Nikon Co., Tokyo, Japan).

Nitrite determination. Nitrite levels were measured using the Griess assay as described previously (26). Nitrite concentrations determined in conditioned supernatants were normalized to total protein concentrations in the cell monolayer. In preliminary experiments, we measured both nitrite and nitrate production (the latter by enzymatically reducing nitrate to nitrite using nitrate reductase). Nitrate levels were approximately $30 \%$ of nitrite levels, and these proportions remained highly consistent between experiments. We therefore chose to measure nitrite alone to confirm NOS activity and NO production.

Immunoblot. nNOS was detected by immunoblot with commercially available anti-nNOS (Transduction Laboratories). In brief, freshly isolated cells or those in culture 
were washed twice with PBS and lysed with a solution containing $1 \%$ SDS, $1.0 \mathrm{mM}$ sodium vanadate, and 10 $\mathrm{mM}$ Tris ( $\mathrm{pH} 7.4)$. Proteins ( $25 \mu \mathrm{g}$ total) were separated by SDS-PAGE and transferred to nitrocellulose. The membrane was incubated in 5\% nonfat milk and then with anti-nNOS for 1 hour at room temperature. nNOS was detected with anti-mouse IgG horseradish peroxidase-conjugated antibody followed by chemiluminescence (ECL Western blotting analysis system; Amersham Life Sciences, Arlington Heights, Illinois, USA). Bands were viewed on multiple exposures to autoradiography film (Eastman Kodak Co., Rochester, New York, USA), and data were collected over a narrow range of $x$-ray film linearity and quantitated by scanning densitometry.

Stellate cell contractility. Stellate cell contractility was quantitated as described previously (9). Briefly, culture vessels were preincubated with PBS containing $1 \%$ BSA for at least 1 hour at $37^{\circ} \mathrm{C}$, then washed twice with PBS and air dried. A combination of Vitrogen (Celltrix Co., Santa Clara, California, USA), 10× MEM (GIBCO BRL) and 0.2 M HEPES (final collagen concentration of approximately $2.4 \mathrm{mg} / \mathrm{mL}$ ) is mixed at $4{ }^{\circ} \mathrm{C}$. After the solution hardened, cell suspensions were layered on top of formed lattices in standard culture medium. After a specified duration in culture, serum-free conditions are introduced, contractile mediators added, and collagen lattices containing cells are detached by gentle circumferential dislodgment of the lattice using a micropipette tip. Contraction was monitored as change in lattice diameter (which is used to calculate area) over time.

Portal pressure and isolated liver perfusion. Portal pressure was measured in vivo by cannulating the portal vein with a polyethylene catheter (PE-50) and recorded with a strain gauge transducer. Subsequently, the liver was prepared as described previously and perfused with Krebs solution oxygenated with $95 \% \mathrm{O}_{2} / 5 \% \mathrm{CO}_{2}$ at $37^{\circ} \mathrm{C}(27)$. The flow rate was adjusted to $0,10,20,40$, and 50 $\mathrm{mL} / \mathrm{min}$ in a pseudorandom order and the inlet pressure was set at zero flow $\left(\mathrm{P}_{\mathrm{Q}=0}\right)$. Multiple-point $\mathrm{P}-\mathrm{Q}$ relationships were generated as described elsewhere (28). The slope of the P-Q relationship (Slope $\mathrm{PQR}_{\mathrm{P}}$ ) reflects the flowdependent incremental resistance of the portal system. Statistics. ANOVA or the Student's $t$ test was used for statistical comparisons. Cells from different animals were used in each experiment. For calculation of mean values and statistical variation, $n$ indicates the number of separate experiments. Error bars depict the SEM; absence of error bars indicates that the SEM was less than $1 \%$ of the mean, unless stated otherwise.

\section{Results}

Ad-mediated $n N O S$ expression in hepatocytes, sinusoidal endothelial cells, and stellate cells. We first tested the ability of recombinant Ad vectors to transude hepatocytes, sinusoidal endothelial cells, and stellate cells in vitro.

\section{Figure 1}

Ad-mediated nNOS gene delivery and activity of nNOS. Parenchymal and nonparenchymal cells were prepared as in Methods. After isolation, cells were grown for 48-72 hours under standard conditions and then transduced with Ad. $\beta$-gal or Ad.nNOS as in Methods. In $\mathbf{a}$ and $\mathbf{b}$, cells were transduced with Ad. $\beta$-gal or Ad.nNOS, respectively, and stained for $\mathrm{NADPH}$ diaphorase. In c, the transduction efficiency after exposure to Ad.nNOS was determined by cell counting as in Methods (Ad. $\beta$-gal is used as an additional control). In d, cell lysates ( $25 \mu \mathrm{g}$ total protein) were immunoblotted for nNOS. In e, 24 hours after transduction, culture supernatants were collected and nitrite detected. ${ }^{A} P<0.01$ compared with control or Ad. $\beta$-gal for each cell type $(n=3)$. $\mathrm{H}$, hepatocyte; SEC, sinusoidal endothelial cells; HSC, hepatic stellate cells.
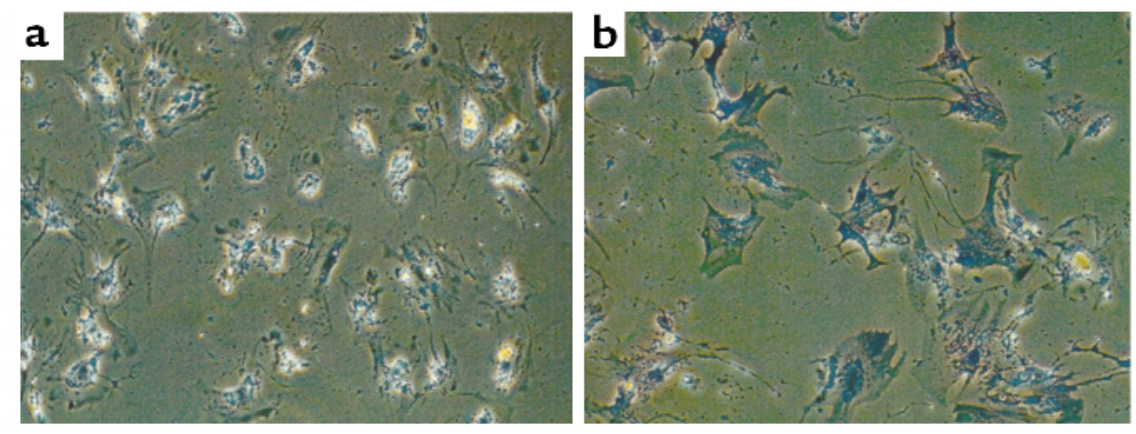

C

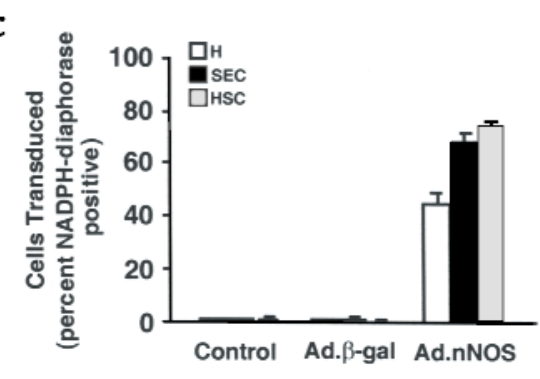

d

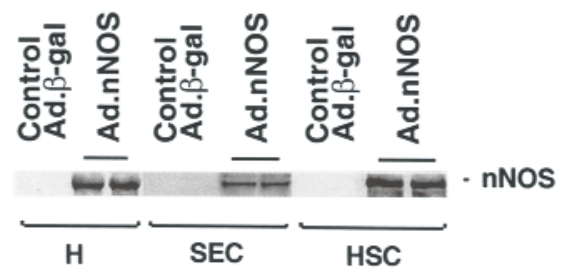

e

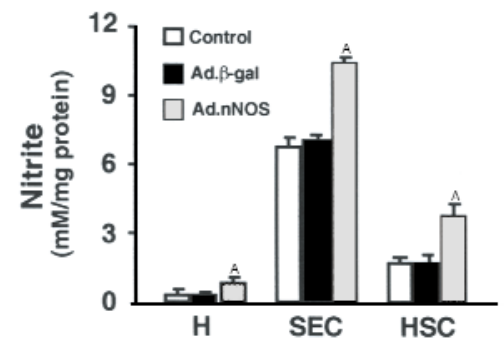


Liver cells were isolated, cultured, and incubated with 1 $\times 10^{9} \mathrm{pfu} / \mathrm{mL}$ Ad. $\beta$-gal. Although approximately $50 \%$ of hepatocytes were transduced at this titer, the transduction efficiency of sinusoidal endothelial and stellate cells was uniformly greater than $70 \%$ (data not shown); as expected, transduction efficiency was directly dependent on Ad titer (data not shown) for all cell types. Given the high-level transduction with Ad. $\beta$-gal at a titer of $10^{9} \mathrm{pfu} / \mathrm{mL}$, we tested the same concentration using the Ad.nNOS construct. Cultured cells were exposed to Ad.nNOS as in Methods, and after 24 hours, nNOS was detected by NADPH diaphorase staining (Figure 1, a-c); although all cell types expressed nNOS as assessed by NADPH diaphorase staining, the proportion of liver cells expressing NOS after transduction cells expressed abundant nNOS as established by immunoblot (Figure 1d). Finally, NOS activity was confirmed by determination of nitrite concentrations in conditioned culture supernatants in hepatocytes and sinusoidal endothelial and stellate cells (Figure 1e).

Ad-mediated nNOS transduction in normal and injured liver in vivo. To determine the effectiveness of in vivo gene transfer of Ad, Ad.nNOS or Ad. $\beta$-gal (same titer) was highest in stellate cells (Figure 1c). Further, stellate

\section{Table 1}

In vivo transduction efficiency of $\mathrm{nNOS}$ in liver cells from normal and injured livers

\begin{tabular}{lccc}
\cline { 2 - 3 } Cell type & Normal & $\mathrm{BDL}$ & $\mathrm{CCl}_{4}$ \\
$\mathrm{H}$ & $41.9 \pm 3.3$ & $39.2 \pm 3.3$ & $37.3 \pm 3.1$ \\
$\mathrm{SEC}$ & $63.1 \pm 7.8$ & $52.7 \pm 4.8^{\mathrm{A}}$ & $41.2 \pm 3.6^{\mathrm{A}}$ \\
$\mathrm{HSC}$ & $65.9 \pm 4.5$ & $50.4 \pm 2.9^{\mathrm{A}}$ & $40.4 \pm 2.7^{\mathrm{A}}$
\end{tabular}

Ad.nNOS was administered via the femoral vein $\left(1.5 \times 10^{11} \mathrm{pfu} / \mathrm{kg}\right)$. For BDL rats, Ad.nNOS was infused 2 days after $\mathrm{BDL}$, and for $\mathrm{CCl}_{4}$ injury, virus was given 2 days after the 10 th dose. Cell harvest was performed 7 days after Ad administration. After cell isolation, cells were allowed to adhere for 18 hours, and NADPH-diaphorase staining was performed as in Methods. Numbers shown represent percent of total NADPH-diaphorase-positive cells (i.e., transduced with nNOS, $n=3$ ). ${ }^{A} P<0.05$ versus normal. $H$, hepatocyte; SEC, sinusoidal endothelial cells; HSC, hepatic stellate cells.

was administered via the femoral vein to normal rats or to those after liver injury (each BDL and $\mathrm{CCl}_{4}$ as in Methods). nNOS expression was identified in the liver by immunohistochemical techniques (Figure 2) as well as by quantitative methods (Figure 3). nNOS was absent in livers transduced with control vector (Figure 2, a and c), whereas nNOS was prominent in cells lining sinusoids (consistent with either sinusoidal endothelial cells or stellate cell expression) and was also
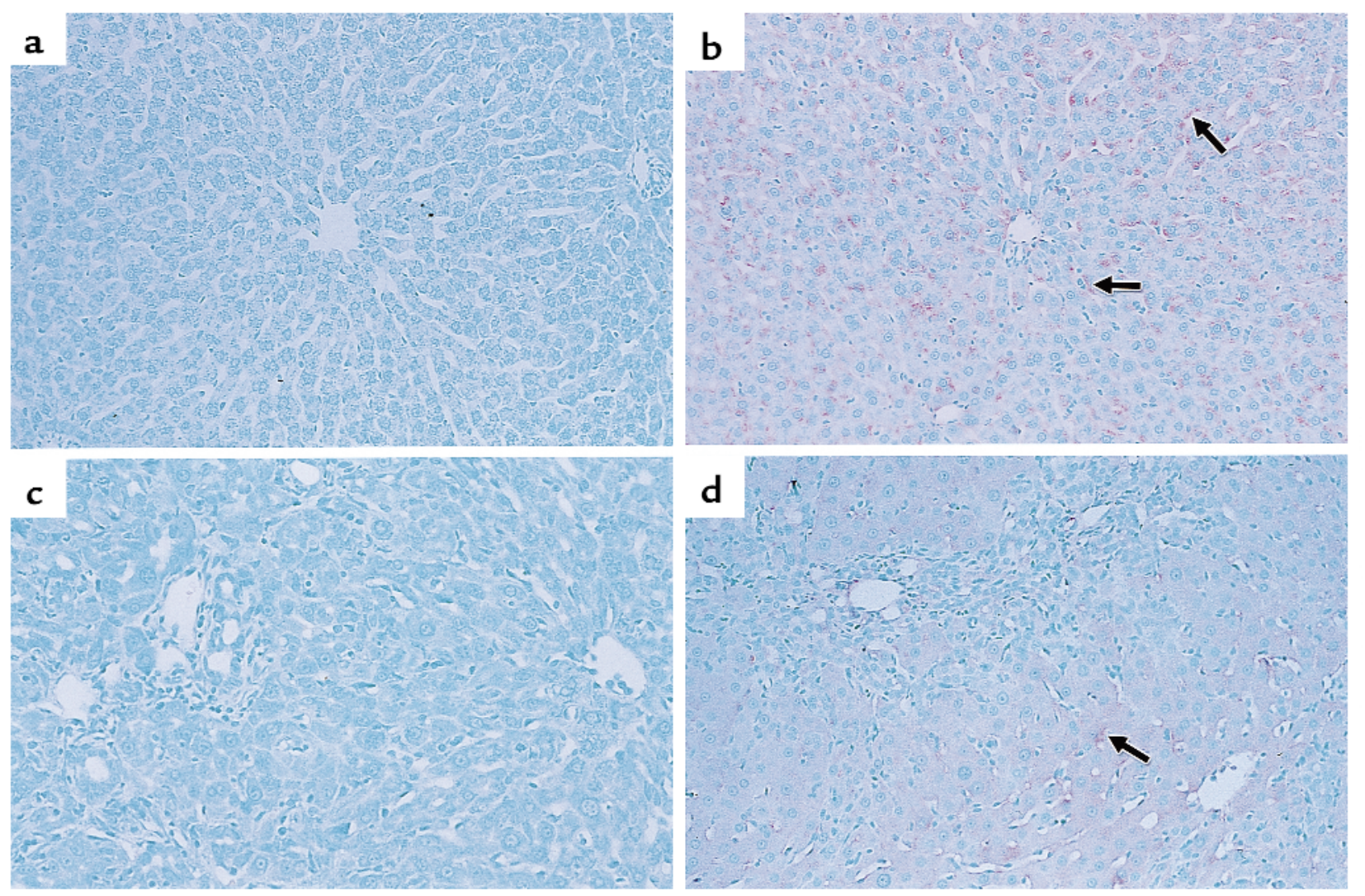

\section{Figure 2}

nNOS expression in vivo in normal and injured liver. Ad $\left(1.5 \times 10^{11} \mathrm{pfu} / \mathrm{kg}\right.$ via femoral vein $)$ was administered to normal rats or those after liver injury as described in Methods. Liver tissue was fixed and probed for $\mathrm{nNOS}$. In controls (a and $\mathbf{c}$ ), immunoreactive nNOS is absent in normal and injured liver (BDL liver is shown; note the prominent bile duct proliferation) transduced with Ad. $\beta$-gal. In contrast, in both normal and injured livers transduced with nNOS ( $\mathbf{b}$ and $\mathbf{d}$ ), immunoreactive nNOS is identified in sinusoidal lining cells (arrows) and in hepatocytes. Immunoreactive $\mathrm{nNOS}$ is less prominent in the injured (d) than in the normal (b) liver. Results were similar for the $\mathrm{BDL}$ and the $\mathrm{CCl}{ }_{4}$ models of injury (the latter, not shown). Data shown are representative of 6 experiments. 
detected in hepatocytes and in vascular smooth muscle cells of portal veins (Figure 2, b and d). The global transduction rate of nNOS in the injured liver was less than that in normal livers (Figure 2, b and d). We more precisely examined nNOS transduction in vivo with Ad.nNOS by isolating cells from each normal and injured rat livers and in vivo gene therapy; nNOS was readily detectable in hepatocytes as well as sinusoidal endothelial and stellate cells (Figure 3, a-c), whether quantitated by immunoblot of isolated cells (Figure 3 , a-c) or counting of NADPH-diaphorase-positive isolated cells (Table 1). Transduction efficiency, as assessed by either method (as well as immunohistochemistry as in Figure 2) was reduced after liver injury, but remained significant. Further, nitrite production, increased in all cell types after each form of liver injury, was less in cells from injured livers than in those from normal liver cells (Table 2). In the BDL model of injury, iNOS can be upregulated to a minor degree in sinusoidal endothelial and stellate cells and can account for increased levels of nitrite in this situation (29). Additionally, basal NOS activity was markedly decreased in sinusoidal endothelial cells after $\mathrm{CCl}_{4}$-induced liver injury, consistent with previous data $(14,15)$. These data indicate that even after severe injury, Ad.nNOS transduced nNOS in injured hepatocytes and sinusoidal endothelial and stellate cells and led to increased NO production compared with control cells.

Transduced $n N O S$ reduces stellate cell contractility. Given the data demonstrating transduction of nNOS in vitro and in vivo by Ad.nNOS, we next asked whether transduced nNOS had biologic effects on liver cells. Initially, we evaluated the effect of nNOS-derived NO on stellate cell contractility in an in vitro assay. Stellate cells isolated from normal rats that had undergone spontaneous activation on collagen lattices were exposed to Ad.nNOS. Contraction of culture-activated stellate cells was significantly decreased in Ad.nNOS-transfected cells compared with Ad. $\beta$-gal controls (Figure 4a). Additionally, cells isolated from injured livers transduced with Ad.nNOS in vivo were also less contractile after exposure to endothelin-1 $(2 \mathrm{nM})$ than were those exposed to control Ad. $\beta$-gal (Figure 4b).

To test the possibility that NO produced in a paracrine fashion might also have effects on stellate cells, we transduced normal rats with Ad.nNOS (or as a

b
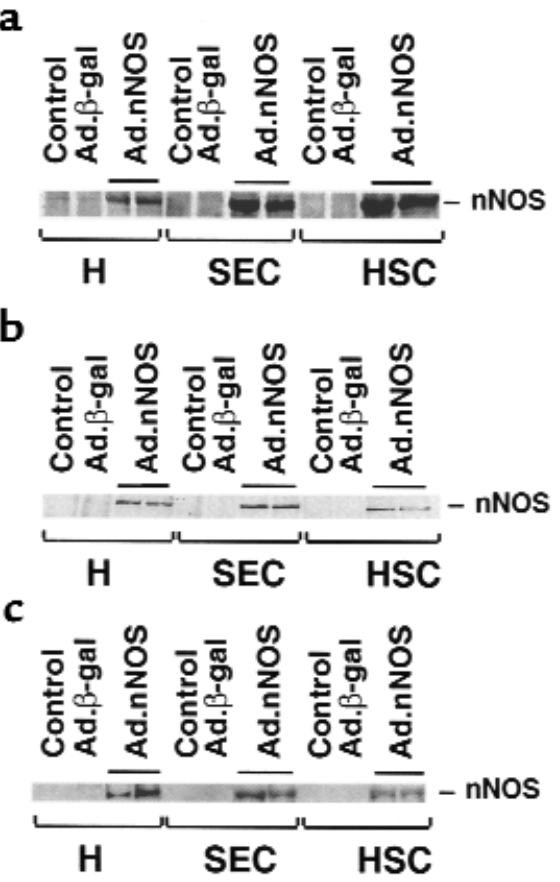

Figure 3

nNOS transduction in vivo in normal and injured hepatic cells. Liver injury (BDL or $\mathrm{CCl}_{4}$ administration) was induced as in Methods. Ad $\left(1.5 \times 10^{11} \mathrm{pfu} / \mathrm{kg}\right.$ via femoral vein $)$ was administered to normal rats or to injured rats 2 days after $\mathrm{BDL}$ or the final dose of $\mathrm{CCl}_{4}$. Hepatocytes, sinusoidal endothelial cells, and stellate cells were isolated 7 days after Ad administration and lysed immediately in sample buffer. Data in a are representative of 4 immunoblots $(25 \mu \mathrm{g}$ total protein) probing cells isolated from normal liver. In $\mathbf{b}$ and $\mathbf{c}$ are shown immunoblots of cells isolated from $\mathrm{BDL}$ and $\mathrm{CCl}_{4}$-treated animals, respectively. $\mathrm{H}$, hepatocyte; SEC, sinusoidal endothelial cells; HSC, hepatic stellate cells.

a control, Ad. $\beta$-gal) and subsequently determined the effect of transduced NO on contractility of activated stellate cells. NO generated from transduced hepatocytes cocultured with activated stellate cells resulted in a 30-40\% decrease in stellate cell contraction (Figure 5). In hepatocyte-stellate cell cocultures exposed to LNMMA $(100 \mu \mathrm{M})$, an inhibitor of NOS, the effect of nNOS was abrogated (data not shown).

Transduced nNOS reduces portal pressure and intrahepatic resistance after cirrhosis and portal hypertension. To investigate the physiological effect of transduced nNOS in the

Table 2

Relative NO (nitrite) production in normal and injured liver cells after in vivo gene transfer

\begin{tabular}{|c|c|c|c|c|c|c|c|c|c|}
\hline \multirow[b]{2}{*}{ Cell type } & \multicolumn{3}{|c|}{ Normal } & \multicolumn{3}{|c|}{ BDL } & \multicolumn{3}{|c|}{$\mathrm{CCl}_{4}$} \\
\hline & Control & Ad. $\beta$-gal & Ad.nNOS & Control & Ad. $\beta$-gal & Ad.nNOS & Control & Ad. $\beta$-gal & Ad.nNOS \\
\hline $\mathrm{H}$ & $0.2 \pm 0.0$ & $0.2 \pm 0.0$ & $1.3 \pm 0.3^{\mathrm{A}}$ & $0.3 \pm 0.0$ & $0.4 \pm 0.0$ & $1.3 \pm 0.3^{\mathrm{A}}$ & $0.2 \pm 0.0$ & $0.2 \pm 0.0$ & $0.3 \pm 0.1^{\mathrm{A}}$ \\
\hline SEC & $5.7 \pm 0.1$ & $5.6 \pm 0.1$ & $8.9 \pm 0.4^{\mathrm{A}}$ & $10.8 \pm 0.2$ & $11.4 \pm 0.3$ & $16.9 \pm 0.5^{\mathrm{A}}$ & $0.4 \pm 0.0$ & $0.4 \pm 0.0$ & $2.1 \pm 0.4^{\mathrm{A}}$ \\
\hline $\mathrm{HSC}$ & $0.4 \pm 0.1$ & $0.4 \pm 0.1$ & $6.2 \pm 0.8^{A}$ & $2.0 \pm 0.5$ & $1.9 \pm 0.3$ & $4.4 \pm 0.8^{\mathrm{A}}$ & $0.3 \pm 0.1$ & $0.3 \pm 0.1$ & $1.3 \pm 0.3^{A}$ \\
\hline
\end{tabular}

Liver injury was induced as in Table 1. Ad. $\beta$-gal or Ad. nNOS was administered via femoral vein $1.5 \times 10^{11} \mathrm{pfu} / \mathrm{kg} 2$ days after BDL or the last dose of CCl . Liver cells were isolated 7 days later and were cultured for 24 hours, after which nitrite concentrations in conditioned supernatants were determined. Shown are means $\pm \mathrm{SEM}\left(\mu \mathrm{M} / \mu \mathrm{g}\right.$ protein). ${ }^{A} P<0.05$ for Ad. nNOS transfected compared with control or Ad. $\beta$-gal-transfected $(n=3$, for each condition). $\mathrm{H}$, hepatocyte; SEC, sinusoidal endothelial cells; HSC, hepatic stellate cells. 
a

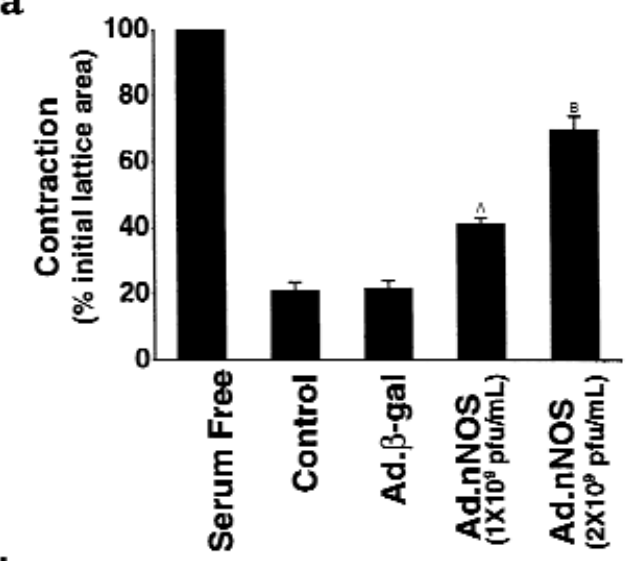

b

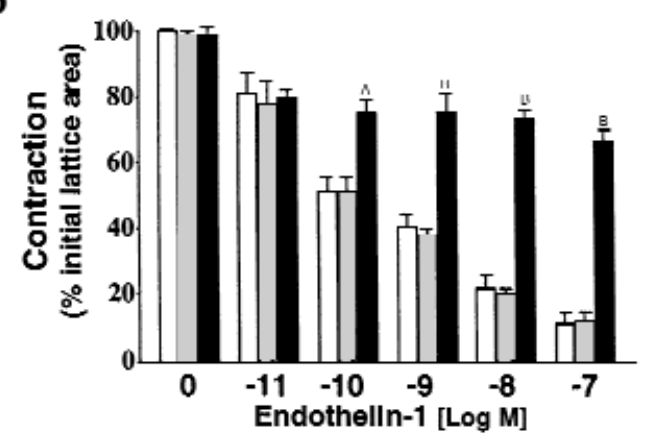

Figure 4

Effect of nNOS gene transfer on stellate cell contractility in vitro and in vivo. In a, the effect of transduced nNOS on stellate cell contractility in vitro was determined. Stellate cells from normal livers were isolated, placed on thick collagen lattices, and allowed to undergo spontaneous activation. Cells were transduced with Ad.nNOS, and 24 hours later contraction assays were performed. In b, BDL was performed and Ad containing nNOS or $\beta$-galactosidase was administered 2 days later; stellate cells were isolated as in Methods and placed on thick collagen matrices. After adherence (for 18 hours), lattices containing cells were placed in serum-free medium, after which endothelin- 1 was added at the indicated concentrations, lattices were detached, and contraction was measured for a further 24 hours. The open bars represent cells from control animals (i.e., vehicle alone); the crossed bars represent cells from rats receiving Ad. $\beta$ gal; and the filled bars depict cells from animals receiving Ad.nNOS. A $P<0.01$ compared with control or Ad. $\beta$-gal $(n=3)$; ${ }^{\text {B }} P<0.001$ compared with control or Ad. $\beta$-gal $(n=3)$.

intact organism, we measured portal pressure and intrahepatic resistance in normal rats and in those after liver injury (either $\mathrm{BDL}$ or $\mathrm{CCl}_{4}$ ). In the $\mathrm{BDL}$ model, Ad.nNOS significantly reduced portal pressure in situ; portal pressure was $13.75 \pm 0.48 \mathrm{~cm} \mathrm{H}_{2} \mathrm{O}$ and $13.98 \pm$ $0.40 \mathrm{~cm} \mathrm{H} \mathrm{H}_{2} \mathrm{O}$ in control and Ad. $\beta$-gal-treated animals, respectively, compared with $12.03 \pm 0.39 \mathrm{~cm} \mathrm{H}_{2} \mathrm{O}$ in Ad.nNOS-treated animals $\left(n=4\right.$; all $1 \times 10^{11} \mathrm{pfu} / \mathrm{kg} ; P<$ 0.05 for control or Ad. $\beta$-gal compared with Ad.nNOS). Further, in the isolated perfused liver, the inlet pressure at zero flow $\left(\mathrm{P}_{\mathrm{Q}=0}\right)$ was reduced in animals exposed to Ad.nNOS compared with either control or Ad. $\beta$-gal animals (Table 3). Similarly, in the $\mathrm{CCl}_{4}$ model of cirrhosis and portal hypertension, portal pressure in situ was reduced from $16.1 \pm 0.4 \mathrm{cmH}_{2} \mathrm{O}$ and $16.0 \pm 0.2 \mathrm{cmH}_{2} \mathrm{O}$ (control and Ad. $\beta$-gal, respectively) to $13.3 \pm 0.2 \mathrm{cmH}_{2} \mathrm{O}$ (Ad.nNOS) $(n=3)$. For this model, in the isolated perfused liver, $\mathrm{P}_{\mathrm{Q}=0}$ was reduced in Ad.nNOS-exposed animals compared with either control or Ad. $\beta$-gal-exposed animals, whereas Slope $e_{\mathrm{PQR}}$ was not significantly altered by nNOS transduction (Table 3 ). Because changes in Slope $_{\mathrm{PQR}}$ reflect predominantly presinusoidal events, and changes in $\mathrm{P}_{\mathrm{Q}=0}$ reflect sinusoidal events (28), the data suggest that the effects of transduced Ad.nNOS are likely to be at the level of the sinusoid. Finally, to establish that the effect of nNOS was due to NO production, we examined the effect of 7-NI, a selective nNOS inhibitor (Table 3). As expected, this inhibitor significantly abrogated the effect of Ad.nNOS.

\section{Discussion}

In this study, we have established that Ad leads to gene transfer not only of hepatocytes, as has been established previously $(30,31)$, but also of sinusoidal endothelial cells and stellate cells, the latter consistent with a previous report that demonstrated efficient in vitro transduction of cultured stellate cells (32). Moreover, we found that the transduction efficiency of sinusoidal endothelial and stellate cells in vivo was high. Even though this result has not been described previously, we believe it is predictable, as hematogenous compounds must traverse the sinusoidal endothelium as well as the sinusoidal space of Disse (wherein reside stellate cells) before coming into contact with hepatocytes. In addition, Ad transduced all cell types in vivo even after profound liver injury $(17,18)$. Although the transduction efficiency of each hepatocyte and nonparenchymal cells was reduced after injury, it remained of high magnitude and resulted in prominent NOS activity in the injured liver. This finding has important implications for gene therapy in diseased liver as well as in other parenchymal organs that have been injured.

In endothelial cells, ecNOS must undergo extensive post-translational processing including myristolation and disassociation from caveolae in order to be functionally active (33-35). Because of the potential of a post-translational handling defect in ecNOS after liver injury $(14,16)$, we chose to transduce a heterologous NOS isoform, nNOS, to sinusoidal endothelial cells (and other liver cells). This resulted in significant production of $\mathrm{NO}$ and important effects on stellate cells individually as well as on the whole liver in an important physiological manner. That this occurred even in injured endothelial cells, despite a presumed defect in post-translational handling of ecNOS, is consistent with a lack of a requirement for extensive post-translational nNOS modification (36).

The use of nNOS in our experiments is significant for several additional reasons. First, it has established a "vasorelaxing" effect with a nonendothelial cell NOS isoform in the liver and therefore has important therapeutic implications for endothelial disorders in which ecNOS may be dysfunctional. Second, the data suggest that liver injury does not impair, in endothelial or other 


\section{Figure 5}

Paracrine effect of transduced nNOS on stellate cell contractility. Stellate cells from normal livers were isolated, placed on thick collagen lattices, and allowed to undergo spontaneous activation. Ad $\left(1.5 \times 10^{11} \mathrm{pfu} / \mathrm{kg}\right.$ via femoral vein $)$ containing $\mathrm{nNOS}$ or $\beta$-gal was administered to normal rats as in Methods, and hepatocytes were isolated 4 days later. Hepatocytes were then layered on top of stellate cells and contraction was induced by serum (20\%) 24 hours later. ${ }^{A} P<0.01$ compared with control or Ad. $\beta$-gal $(n=3)$.

cell types, the function of nNOS. Although these data make the use of nNOS an attractive approach in cirrhosis (and for other vascular disorders), we have not excluded the possibility that overexpression of ecNOS could lead to similar effects. Indeed, overexpression of ecNOS in injured endothelial cells is likely to provide important insight into mechanisms of post-translational handling of ecNOS.

Given that an important site of potential regulation of sinusoidal blood flow is at the level of the sinusoid, which is made up of both sinusoidal endothelial cells and perisinusoidal stellate cells, we speculate that the physiological effect of nNOS demonstrated in the whole liver was a result of $\mathrm{NO}$ production by sinusoidal endothelial or perisinusoidal stellate cells (and its likely action on stellate cells). Moreover, we demonstrated relaxing effects of transduced nNOS on highly contractile stellate cells. In addition, our finding in perfusion studies that transduced $\mathrm{nNOS}$ affected primarily $\mathrm{P}_{\mathrm{Q}=0}$ rather than Slope $e_{\mathrm{PQR}}$, suggested that the effect of nNOS was at the level of the sinusoid. Although the effects of transduced nNOS in the whole organ were likely to be in part from due to those on stellate and endothelial cells (i.e., the major components of the hepatic "vascular space"), it is important to emphasize that hepatocytes were also transduced by the Ad.nNOS vector used and that transduced hepatocytes produced NO, which had physiological effects on stellate cells (Figure 5). Further, we did not specifically examine Kupffer cells, which were also likely to be transduced, and which could have produced physiologically active NO. Thus, we cannot

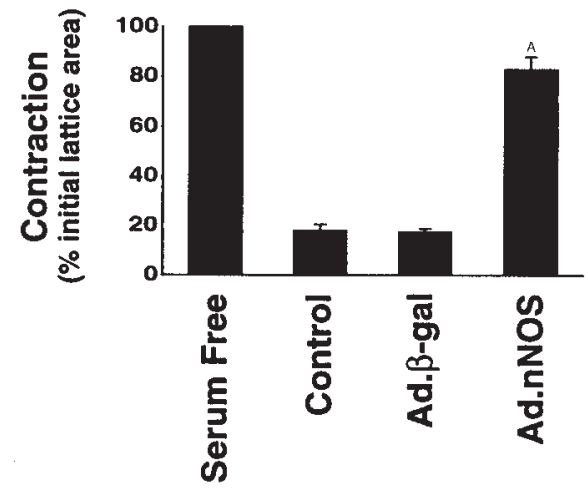

exclude the possibility that hepatocyte or Kupffer cell-generated NO plays a role in the models studied. Finally, we found that some smooth muscle cells in portal vein radicles were transduced (Figure 2); although we cannot exclude the possibility that transduced nNOS had effects on smooth muscle cells within the liver, which in turn contributed to the demonstrated reduction in portal pressure and intrahepatic resistance, the major effect of transduced nNOS appeared to be at the level of the sinusoid (based on the lack of a change in Slope $\left._{\mathrm{PQR}}\right)$. Further studies are under way to explore the precise anatomic site in the liver at which transduced nNOS may have its physiological effects.

The results presented in this study are consistent with previous data indicating that nitrogenous compounds are capable of affecting portal vascular resistance (37). From a therapeutic standpoint, however, amounts of nitrogenous compounds administered systemically that are required to reduce portal (intrahepatic) resistance are large and often are associated with significant side effects. In contrast, because Ad is preferentially taken up by the liver, Ad vectors provide particularly favorable vehicles for targeting biologically active agents such as nNOS specifically to the liver. Further, given the data demonstrating uptake of Ad in liver cells even in the presence of significant underlying liver injury, our findings have significant therapeutic implications for

Table 3

Effect of transduced nNOS on portal pressure

erfusion pressure $\left(\mathrm{cmH}_{2} \mathrm{O}\right)$

\begin{tabular}{|c|c|c|c|c|c|c|}
\hline \multirow[b]{2}{*}{ Transduction state } & \multicolumn{4}{|c|}{ Flow rate $(\mathrm{mL} / \mathrm{min})$} & \multirow{2}{*}{$\begin{array}{c}\mathrm{P}_{\mathrm{Q}=0} \\
\left(\mathrm{~cm} \mathrm{H}_{2} \mathrm{O}\right)\end{array}$} & \multirow{2}{*}{$\begin{array}{c}\text { Slope }_{\mathrm{PQR}} \\
\left(\mathrm{cmH}_{2} \mathrm{O} \cdot \mathrm{min}^{\left.-\mathrm{ml}^{-1}\right)}\right)\end{array}$} \\
\hline & 20 & 30 & 40 & 50 & & \\
\hline Normal & $4.1 \pm 0.2$ & $5.6 \pm 0.4$ & $7.4 \pm 0.7$ & $10.4 \pm 0.5$ & $0.81 \pm 0.10$ & $0.15 \pm 0.01$ \\
\hline \multicolumn{7}{|l|}{$\mathrm{CCl}_{4}$} \\
\hline Ad. $\beta$-gal & $8.5 \pm 0.5^{\mathrm{A}}$ & $11.1 \pm 0.9^{\mathrm{A}}$ & $14.7 \pm 0.1^{\mathrm{A}}$ & $16.9 \pm 0.3^{A}$ & $2.98 \pm 0.13^{A}$ & $0.28 \pm 0.02^{\mathrm{A}}$ \\
\hline Ad.nNOS & $6.3 \pm 0.2^{A, B}$ & $8.7 \pm 0.3^{\mathrm{A}, \mathrm{B}}$ & $12.0 \pm 0.2^{\mathrm{A}, \mathrm{B}}$ & $14.9 \pm 0.4^{\mathrm{A}, \mathrm{B}}$ & $1.16 \pm 0.03^{\mathrm{A}, \mathrm{B}}$ & $0.27 \pm 0.01^{\mathrm{A}}$ \\
\hline \multicolumn{7}{|l|}{ BDL } \\
\hline Ad. $\beta$-gal & $8.8 \pm 0.1^{\mathrm{A}}$ & $11.0 \pm 0.6^{A}$ & $13.6 \pm 0.5^{\mathrm{A}}$ & $15.4 \pm 0.6^{A}$ & $3.32 \pm 0.17^{A}$ & $0.25 \pm 0.02^{\mathrm{A}}$ \\
\hline Ad.nNOS & $7.4 \pm 0.1^{\mathrm{A}, \mathrm{B}}$ & $9.0 \pm 0.2^{\mathrm{A}, \mathrm{B}}$ & $11.2 \pm 0.4^{\mathrm{A}, \mathrm{B}}$ & $13.9 \pm 0.4^{\mathrm{A}, \mathrm{B}}$ & $2.00 \pm 0.10^{\mathrm{A}, \mathrm{B}}$ & $0.24 \pm 0.01^{\mathrm{A}}$ \\
\hline $7-\mathrm{NI}$ & $9.1 \pm 0.1^{\mathrm{A}}$ & $11.9 \pm 0.2^{\mathrm{A}}$ & $13.8 \pm 0.1^{\mathrm{A}}$ & $16.2 \pm 0.2^{\mathrm{A}}$ & $3.33 \pm 0.22^{\mathrm{A}}$ & $0.27 \pm 0.02^{\mathrm{A}}$ \\
\hline 7-NI/Ad.nNOS & $9.0 \pm 0.3^{\mathrm{A}, \mathrm{C}}$ & $11.6 \pm 0.2^{\mathrm{A}, \mathrm{C}}$ & $13.5 \pm 0.3^{\mathrm{A}, \mathrm{C}}$ & $15.8 \pm 0.5^{\mathrm{A}, \mathrm{C}}$ & $3.24 \pm 0.10^{A, C}$ & $0.26 \pm 0.05^{\mathrm{A}}$ \\
\hline
\end{tabular}

Liver injury (BDL or $\mathrm{CCl}_{4}$ ) was as in Methods. Either Ad.nNOS or Ad. $\beta$-gal (each $1.5 \times 10^{11} \mathrm{pfu} / \mathrm{kg}$ ) was injected via the femoral vein 7 days before isolated liver perfusion as in Methods. Portal pressure, monitored continuously, was recorded at incremental flow rates, and portal resistance was calculated as in Methods. In experiments in which $7 \mathrm{NI}$ was used, this compound was administered by intraperitoneal injection 1 day before BDL ( $25 \mathrm{mg} / \mathrm{kg})$ and every 2 days thereafter. ${ }^{A} P<0.05$ vs. normal. ${ }^{B} P<0.05$ vs. Ad. $\beta$-gal for each injury model. ${ }^{C} P<0.05$ vs. Ad. nNOS (all, $\left.n=4\right)$. 
patients with cirrhosis. Although we did not detect significant hepatocellular toxicity at the concentration of Ad used in our in vivo experiments, potential toxicity remains an important concern, particularly in humans.

The endothelium plays a central role in the local regulation of vascular tone (38-40). Mounting evidence suggests that secondary endothelial dysfunction is an important component of many forms of vascular disease, including in such states as congestive heart failure and hypertension (41). In pathological states, in particular, an imbalance between ET-1 and EDRF (NO) appears to be prominent (42). Further, we and others have demonstrated that a similar situation exists in the cirrhotic liver $(5,13,15)$, with enhanced ET-1 production and reduced NO production. Given that insufficient NO production appears to be a key common denominator of many of these forms of endothelial dysfunction $(43,44)$, the data presented in this study have fundamental implications for treatment of a wide variety of diseases in which endothelial dysfunction resulting in a defect in $\mathrm{NO}$ production plays a role.

\section{Acknowledgments}

This work was supported by grants from the National Institutes of Health (DK 57830 and DK 50574) and the American Digestive Health Foundation (Fiterman Basic Research Award).

1. Friedman, S.L. 1993. Seminars in medicine of the Beth Israel Hospital, Boston. The cellular basis of hepatic fibrosis. Mechanisms and treatment strategies. N. Engl. J. Med. 328:1828-1835.

2. Shah, V., Garcia-Cardena, G., Sessa, W.C., and Groszmann, R.J. 1998. The hepatic circulation in health and disease: report of a single-topic symposium. Hepatology. 27:279-288.

3. Bauer, M., Zhang, J.X., Bauer, I., and Clemens, M.G. 1994. ET-1 induced alterations of hepatic microcirculation: sinusoidal and extrasinusoidal sites of action. Am. J. Physiol. 267:G143-G149.

4. Zhang, J.X., Pegoli, W.J., and Clemens, M.G. 1994. Endothelin-1 induces direct constriction of hepatic sinusoids. Am. J. Physiol. 266:G624-G632.

5. Bauer, M., et al. 1995. Chronic ethanol consumption increases hepatic sinusoidal contractile response to endothelin-1 in the rat. Hepatology. 22:1565-1576.

6. Okumura, S., et al. 1994. Vasoactive effect of endothelin-1 on rat liver in vivo. Hepatology. 19:155-161.

7. Suematsu, M., et al. 1995. Carbon monoxide: an endogenous modulator of sinusoidal tone in the perfused rat liver. J. Clin. Invest. 96:2431-2437

8. Tanaka, Y., et al. 1991. Phenotypic modulation in lipocytes in experimental liver fibrosis. J. Pathol. 164:273-278.

9. Rockey, D.C., Housset, C.N., and Friedman, S.L. 1993. Activationdependent contractility of rat hepatic lipocytes in culture and in vivo. J. Clin. Invest. 92:1795-1804.

10. Pinzani, M., et al. 1996. Endothelin 1 is overexpressed in human cirrhotic liver and exerts multiple effects on activated hepatic stellate cells. Gastroenterology. 110:534-548.

11. Gandhi, C.R., Sproat, L.A., and Subbotin, V.M. 1996. Increased hepatic endothelin-1 levels and endothelin receptor density in cirrhotic rats. Life Sci. 58:55-62.

12. Rockey, D.C., et al. 1998. Cellular localization of endothelin-1 and increased production in liver injury in the rat: potential for autocrine and paracrine effects on stellate cells. Hepatology. 27:472-480.

13. Shah, V., et al. 1997. Liver sinusoidal endothelial cells are responsible for nitric oxide modulation of resistance in the hepatic sinusoids. $J$. Clin. Invest. 100:2923-2930.

14. Rockey, D.C., and Chung, J.J. 1998. Reduced nitric oxide production by endothelial cells in cirrhotic rat liver: endothelial dysfunction in portal hypertension. Gastroenterology. 114:344-351.

15. Gupta, T.K., Toruner, M., Chung, M.K., and Groszmann, R.J. 1998. Endothelial dysfunction and decreased production of nitric oxide in the intrahepatic microcirculation of cirrhotic rats. Hepatology.
28:926-931.

16. Shah, V., et al. 1999. Impaired endothelial nitric oxide synthase activity associated with enhanced caveolin binding in experimental cirrhosis in the rat. Gastroenterology. 117:1222-1228.

17. Kountouras, J., Billing, B.H., and Scheuer, P.J. 1984. Prolonged bile duct obstruction: a new experimental model for cirrhosis in the rat. Br. J. Exp. Pathol. 65:305-311.

18. Proctor, E., and Chatamra, K. 1982. High yield micronodular cirrhosis in the rat. Gastroenterology. 83:1183-1190.

19. Bissell, D.M., Hammaker, L.E., and Meyer, U.A. 1973. Parenchymal cells from adult rat liver in nonproliferating monolayer culture. I. Functional studies. J. Cell Biol. 59:722-734.

20. de Leeuw, A.M., McCarthy, S.P., Geerts, A., and Knook, D.L. 1984. Purified rat liver fat-storing cells in culture divide and contain collagen. Hepatology. 4:392-403.

21. Friedman, S.L., and Roll, F.J. 1987. Isolation and culture of hepatic lipocytes, Kupffer cells, and sinusoidal endothelial cells by density gradient centrifugation with Stractan. Anal. Biochem. 161:207-218.

22. Irving, M.G., Roll, F.J., Huang, S., and Bissell, D.M. 1984. Characterization and culture of sinusoidal endothelium from normal rat liver: lipoprotein uptake and collagen phenotype. Gastroenterology. 87:1233-1247.

23. Itzhak, Y., Martin, J.L., Black, M.D., and Huang, P.L. 1998. The role of neuronal nitric oxide synthase in cocaine-induced conditioned place preference. Neuroreport. 9:2485-2488.

24. Channon, K.M., Blazing, M.A., Shetty, G.A., Potts, K.E., and George, S.E. 1996. Adenoviral gene transfer of nitric oxide synthase: high level expression in human vascular cells. Cardiovasc. Res. 32:962-972.

25. Chao, D.S., Hwang, P.M., Huang, F., and Bredt, D.S. 1996. Localization of neuronal nitric oxide synthase. Methods Enzymol. 268:488-496.

26. Green, L.C., et al. 1982. Analysis of nitrate, nitrite, and [15N]nitrate in biological fluids. Anal. Biochem. 126:131-138.

27. Weisiger, R.A., Fitz, J.G., and Scharschmidt, B.F. 1989. Hepatic oleate uptake. Electrochemical driving forces in intact rat liver. J. Clin. Invest. 83:411-420.

28. Pannen, B.H., Bauer, M., Zhang, J.X., Robotham, J.L., and Clemens, M.G. 1996. A time-dependent balance between endothelins and nitric oxide regulating portal resistance after endotoxin. Am. J. Physiol. 271:H1953-H1961.

29. Rockey, D.C., and Chung, J.J. 1997. Regulation of inducible nitric oxide synthase and nitric oxide during hepatic injury and fibrogenesis. Am. J. Physiol. 273:G124-G130.

30. Kovesdi, I., Brough, D.E., Bruder, J.T., and Wickham, T.J. 1997. Adenoviral vectors for gene transfer. Curr. Opin. Biotechnol. 8:583-589.

31. Maher, J.J., Scott, M.K., Saito, J.M., and Burton, M.C. 1997. Adenovirus-mediated expression of cytokine-induced neutrophil chemoattractant in rat liver induces a neutrophilic hepatitis. Hepatology. 25:624-630.

32. Hellerbrand, C., et al. 1998. Inhibition of NFkappaB in activated rat hepatic stellate cells by proteasome inhibitors and an IkappaB superrepressor. Hepatology. 27:1285-1295.

33. Feron, O., Michel, J.B., Sase, K., and Michel, T. 1998. Dynamic regulation of endothelial nitric oxide synthase: complementary roles of dual acylation and caveolin interactions. Biochemistry. 37:193-200.

34. Feron, O., Saldana, F., Michel, J.B., and Michel, T. 1998. The endothelial nitric-oxide synthase-caveolin regulatory cycle. J. Biol. Chem. 273:3125-3128.

35. Liu, J., Hughes, T.E., and Sessa, W.C. 1997. The first 35 amino acids and fatty acylation sites determine the molecular targeting of endothelial nitric oxide synthase into the Golgi region of cells: a green fluorescent protein study. J. Cell Biol. 137:1525-1535.

36. Bredt, D.S. 1996. Targeting nitric oxide to its targets. Proc. Soc. Exp. Biol. Med. 211:41-48.

37. Blei, A.T., and Gottstein, J. 1986. Isosorbide dinitrate in experimental portal hypertension: a study of factors that modulate the hemodynamic response. Hepatology. 6:107-111.

38. Yanagisawa, M. 1994. The endothelin system. A new target for therapeutic intervention. Circulation. 89:1320-1322.

39. Rubanyi, G.M. 1993. The role of endothelium in cardiovascular homeostasis and diseases. J. Cardiovasc. Pharmacol. 22(Suppl 4):S1-14.

40. Rudic, R.D., et al. 1998. Direct evidence for the importance of endothelium-derived nitric oxide in vascular remodeling. J. Clin. Invest. 101:731-736.

41. Boulanger, C.M. 1999. Secondary endothelial dysfunction: hypertension and heart failure. J. Mol. Cell. Cardiol. 31:39-49.

42. Drexler, H., and Hornig, B. 1999. Endothelial dysfunction in human disease. J. Mol. Cell. Cardiol. 31:51-60.

43. Mombouli, J.V., and Vanhoutte, P.M. 1999. Endothelial dysfunction: from physiology to therapy. J. Mol. Cell. Cardiol. 31:61-74.

44. Michel, T., and Feron, O. 1997. Nitric oxide synthases: which, where, how, and why? J. Clin. Invest. 100:2146-2152. 